\title{
Editorial
}

\section{Neck dissection: historical perspective}

\author{
Alfio Ferlito, M.D., F.R.C.S. (Ed.), F.R.C.S., F.A.S.C.P., F.R.C.S. (GlasG.), \\ K. Thomas Robbins, M.D., F.A.C.S., F.R.C.S.C.*, Alessandra Rinaldo, M.D.
}

Because of the proclivity of head and neck cancer to metastasize to the regional lymph nodes, a discussion of the management, regardless of the primary sites, mandates a concurrent discussion on how to treat the neck. With the exception of distant metastases the most adverse independent prognostic factor in squamous carcinoma of the head and neck is the presence of involved cervical lymph nodes. ${ }^{1}$ Furthermore, metastatic cancer involving the cervical lymph nodes is the most common pattern of recurrence in patients with head and neck squamous carcinoma. Such an event generally carries a fatal prognosis. ${ }^{2}$ The presence of a single involved lymph node reduces survival by 50 per cent. ${ }^{3}$ Contralateral or bilateral cervical disease reduces prognosis by an additional 50 per cent. Therefore, careful consideration must be given to treatment of the neck. Neck dissection has been used in the treatment of cervical metastases and it is generally accepted that, if the risk of lymph node metastases in the neck is greater than 20 per cent, elective treatment is required.

Without diminishing the importance of the contributions by some pioneers, the first systematic anatomical description of en bloc dissection of the neck was published in English by George Washington Crile, Sr, from Cleveland Clinic, Ohio, in the Transactions of the Southern Surgical and Gynecological Association in $1905 .{ }^{4}$ This landmark article was entitled 'On the surgical treatment of cancer of the head and neck. With a summary of one hundred and twenty-one operations performed upon one hundred and five patients' and is composed of 20 pages. His paper on this topic is accompanied by 12 drawings of great clarity and a discussion of nine pages. In particular, Charles H. Mayo of Rochester in the discussion stated 'A large part of abdominal work is recreation as compared with the bulk of what might be called the heavy surgery of the neck, which Dr Crile has so well described'. This publication is often not mentioned in the medical literature, however, this report represents a monumental piece of work in the treatment of the neck metastases. The operation he described has come to be known as radical neck dissection. He advocated a block resection of the cervical lymph nodebearing tissue to be removed either in continuity with the primary cancer or as a secondary operation for subsequent metastases. The surgical procedure as originally conceived by $\mathrm{Crile}^{4}$ is accomplished by sacrificing the sternocleidomastoid muscle, the internal jugular vein, the submaxillary gland and the omoyhyoid muscle. Crile ${ }^{4}$ mentioned a study of 4500 cases with head and neck cancer made for him by $\mathrm{Dr}$ Hitchings in which fewer than one per cent of the tumours of the head and neck had secondary foci in distant tissue or organs (but higher rates of distant metastases have been reported in more recent studies). ${ }^{5}$ From this study Crile ${ }^{4}$ concluded that if the neck lymphatics could be removed en bloc, more cures could be accomplished. He was considered the primary proponent of this type of surgery in the United States. Crile ${ }^{4}$ stated that the key of dissemination of the cancer was the internal jugular vein, and therefore it was imperative to sacrifice the vein by a block dissection. Crile ${ }^{4}$ practised composite block dissections with removal of a part of the mandible for lesions of the tongue and floor of the mouth.

In 1906, Crile ${ }^{6}$ published another paper on block dissection in the Journal of the American Medical Association. This second publication is always quoted as the original approach to neck dissection and is entitled 'Excision of cancer of the head and neck. With a special reference to the plan of dissection based on one hundred and thirty-two operations'. This paper is also accompanied by drawings of great clarity (the illustrations are the same as published in the previous paper by Crile). With these papers Crile put block dissection on a par with the Halsted operation for the treatment of breast cancer. ${ }^{4,6}$ Almost all clinicians mention the second Crile paper ${ }^{6}$ as the original description of neck dissection, while ignoring the first publication. This error was later constantly copied in the medical literature and illustrates well the dangers of quoting historical references secondhand, particularly when the original paper was in another language. The mistake was also reported in historical landmarks in head and neck cancer surgery articles. In his second publication he reported the results of treatment of 132 head and neck cancers. Crile ${ }^{6}$ noted that among 48 patients who did not have a radical neck 
dissection, only nine were alive three years later; while among 12 other patients who underwent radical neck dissection, three years after the surgical procedure, nine were alive. From these data, he concluded that the radical neck block dissection was four times more effective than the less radical. The radical block operation has stood the test of time and has saved countless lives. ${ }^{7}$ The only real difference between the two publications by Crile $^{4,6}$ relates to the number of the operations: 121 and 132, respectively.

Crile ${ }^{4,6}$ mentioned that the spinal accessory nerve may be preserved if there is no gross tumour near it. It is also interesting to note from the accompanying drawings of the more radical en bloc resections that the spinal accessory nerve and the ansa hypoglossi were preserved. The functional outcome and quality of life are directly related to the sacrifice or preservation of the spinal accessory nerve, while the sternocleidomastoid muscle or jugular vein rarely cause major functional or cosmetic issues. He removed the submaxillary gland only when the cervical nodes were found to be involved. When lymph nodes were not palpated prior to the operation but, after opening the neck a single involved lymph node of small size was found, the dissection was made to include a part of the sternocleidomastoid muscle. Conceptually, Crile must have given thought to a modified neck dissection with preservation of the spinal accessory nerve. ${ }^{4,6}$

Crile is often referred to as the grandfather of radical neck dissection, but actually he is also the grandfather of neck dissection in its various modified forms. He suspected a biological difference in tumour behaviour and prognosis between patients who had palpable suspicious neck nodes and those who did not. He favoured a more aggressive surgical treatment (extensive dissection) for those who had palpable disease and more limited operation for the others. He believed that neck dissection in early cases in the absence of palpable disease was important. '. . a dissection is indicated whether the glands are or are not palpable. Palpable glands may be inflammatory and impalpable glands may be carcinomatous. A strict rule of excision should therefore be followed ${ }^{4}$ Increased rates of cure and decreased rates of recurrence occurred if the clinically negative neck was treated at the time of primary surgery. Crile, ${ }^{4}$ therefore, advised elective neck dissection in some situations for $\mathrm{N}_{0}$-staged neoplastic disease. In 1901, Jacob Da Silva SolisCohen of Philadelphia, America's first head and neck surgeon, mentioned the necessity of removing the lymphatics of the neck during total laryngectomy regardless of whether there was clinical evidence of cancer in them. ${ }^{8}$ In 1885 , Butlin ${ }^{9}$ also advocated elective removal of cervical lymph nodes for tongue cancer.

From 1900, Crile ${ }^{4,6}$ performed different types of neck dissections and using the current proposed terminology by the American Head and Neck Society and the American Academy of Otolaryngo-
logy-Head and Neck Surgery, ${ }^{10}$ it can be said that he developed radical neck dissection, modified radical neck dissection, selective neck dissection and recommended elective (prophylactic) neck dissection in the non-palpable neck. In particular $\mathrm{Crile}^{4}$ advised elective neck dissection for cancer of the lip. This practice was given up later by surgeons. Crile ${ }^{4}$ recommended preservation of the internal jugular vein, sternocleidomastoid muscle and spinal accessory nerve in patients without palpable lymph nodes. Furthermore, he suggested removal only when the regional lymph nodes were known to drain the field of the original focus of the disease even when metastases could not be demonstrated. Weir ${ }^{11}$ in his book on Otolaryngology stated 'He (Crile) developed "partial" and "radical" neck dissection operations which involved removal of the primary tumour en bloc with the neck dissection. Although the operation was taken up throughout the world there was a lull in interest until the early 1950s'.

In 1933, Blair and Brown ${ }^{12}$ advocated the routine removal of the spinal accessory nerve in neck dissection to decrease operating time and, more importantly, increase the certainty of total neck node removal of the cervical lymph nodes.

In 1951, Martin et al. ${ }^{13}$ from the Memorial Hospital published in Cancer an analysis of 1450 cases of neck dissection and categorically insisted that the spinal accessory nerve, internal jugular vein and the sternocleidomastoid muscle should be removed in the presence of cervical lymph node metastasis. Besides, they categorically stated 'Any technique that is designed to preserve the spinal accessory nerve should be condemned unequivocally'. The technical precepts described by Martin et al. ${ }^{13}$ were followed by many American surgeons until the latter part of the 20th century when modifications in technique began to find acceptance. The greatest impetus to the development of radical neck dissection, including the removal of all the lymph nodes from levels I through $\mathrm{V}$ together with the spinal accessory nerve, the internal jugular vein, the sternocleidomastoid muscle, the omohyoid muscle, the submandibular gland, the tail of the parotid gland, and the cervical plexus nerves, for treatment of head and neck cancer came from Martin and his colleagues. ${ }^{13}$ They $^{13}$ believed that the 'routine prophylactic neck dissection is considered illogical and unacceptable' for cancer of the oral cavity. Conley ${ }^{14}$ was another strong proponent of radical neck dissection.

Historically, the treatment of patients with clinically palpable metastatic disease (overt nodal disease) in the neck has been radical neck dissection. This surgical procedure remained unchallenged until the late 1960s. Due to its significant morbidity, from 1952 Suárez continued to work using a type of technique that enabled him to completely eliminate the lymph node tissue in the neck along with the primary tumour, while carefully preserving the spinal accessory nerve, the sternocleidomastoid muscle and the internal jugular vein. ${ }^{15}$ This surgical technique is called functional neck dissection and is a safe 
surgical approach to the treatment of non-palpable and palpable mobile lymph nodes in patients with head and neck cancer. ${ }^{16}$ It is not a modification of the radical neck dissection but a different surgical procedure based conceptually on the fascial compartments of the neck. ${ }^{17}$ Osvaldo Suárez is the oftenforgotten father of functional neck dissection in the non-Spanish-speaking literature. ${ }^{18}$ Selective neck dissections, surgically targeting regional nodal groups at risk for metastasis based on patterns of predictability, were popularized by head and neck surgeons at the M.D. Anderson Cancer Center in Houston, TX, based on Ballantyne's approach. ${ }^{19}$ These surgical procedures evolved based on the pioneering work of Suárez and, in essence, represent modifications of functional neck dissections through improved knowledge of the patterns of spread to the cervical lymph nodes from head and neck cancers.

\section{References}

1 Ferlito A, Rinaldo A, Robbins KT, Leemans CR, Shah JP, Shaha AR, et al. Changing concepts in the surgical management of the cervical node metastasis. Oral Oncol 2003;39:429-35

2 Hosal AS. Is radical neck dissection the only surgical option for the $\mathrm{N}+$ neck? Proceedings of the 5th International Conference on Head and Neck Cancer. San Francisco: 2000;457-62

3 Myers EN, Fagan JJ. Treatment of the $\mathrm{N}+$ neck in squamous cell carcinoma of the upper aerodigestive tract. Otolaryngol Clin North Am 1998;31:671-86

4 Crile GW. On the surgical treatment of cancer of the head and neck. With a summary of one hundred and twenty-one operations performed upon one hundred and five patients. Trans South Surg Gynecol Assoc 1905;18:108-27

5 Ferlito A. Distant metastases from head and neck cancer. A multi-institutional view. ORL J Otorhinolaryngol Relat Spec 2001;63:187-266

6 Crile GW. Excision of cancer of the head and neck. With special reference to the plan of dissection based on one hundred and thirty-two operations. J Am Med Assoc 1906; 47:1780-6

7 Stell PM. The management of cervical lymph nodes in head and neck cancer. Proc R Soc Med 1975;68:83-5
8 Solis-Cohen J. The surgical treatment of laryngeal cancer. Trans Am Laryngol Assoc 1901;22:75-87

9 Butlin HT. Diseases of the Tongue. London: Cassell \& Co., 1885

10 Robbins KT, Clayman G, Levine PA, Medina J, Sessions $\mathrm{R}$, Shaha A, et al. American Head and Neck Society; American Academy of Otolaryngology-Head and Neck Surgery. Neck dissection classification update: revisions proposed by the American Head and Neck Society and the American Academy of Otolaryngology-Head and Neck Surgery. Arch Otolaryngol Head Neck Surg 2002;128:751-8

11 Weir N. Otolaryngology. An Illustrated History. London: Butterworths, 1990

12 Blair VP, Brown JP. The treatment of cancerous or potentially cancerous cervical lymph nodes. Ann Surg 1933;98:650-61

13 Martin HE, Del Valle B, Ehrlich H, Cahan WG. Neck dissection. Cancer 1951;4:441-99

14 Conley J. Radical neck dissection. Laryngoscope 1975;84:1344-52

15 Suárez O. El problema de las metastasis linfáticas y alejadas del cáncer de laringe e hipofaringe. Rev Otorrinolaringol 1963;23:83-99

16 Ferlito A, Gavilán J, Buckley JG, Shaha AR, Miodonski AJ, Rinaldo A. Functional neck dissection: fact and fiction. Head Neck 2001;23:804-8

17 Gavilán J, Herranz J, DeSanto LW, Gavilán C. Functional and Selective Neck Dissection. New York: Thieme, 2002

18 Ferlito A, Rinaldo A. Osvaldo Suárez: often-forgotten father of functional neck dissection (in the non-Spanishspeaking literature). Laryngoscope (in press)

19 Jesse RH, Ballantyne AJ, Larson D. Radical or modified neck dissection: a therapeutic dilemma. Am J Surg 1978;136:516-9

Address for correspondence:

Alfio Ferlito, M.D., F.R.C.S. (Ed.), F.R.C.S., F.A.S.C.P.,

F.R.C.S. (Glasg.),

Director of the Department of Surgical Sciences,

Professor and Chairman of the ENT Clinic,

University of Udine,

Policlinico Universitario,

Piazzale S. Maria della Misericordia,

I-33100 Udine, Italy.

Fax: +390432 559339

E-mail: a.ferlito@uniud.it 